\title{
Germanica
}

GERMANICA $\quad 10 \mid 1992$

Mosaïques littéraires

\section{La traduction, art second}

Die Kunst des Übersetzers

Erika Tunner

\section{(2) OpenEdition}

Journals

Édition électronique

URL : http://journals.openedition.org/germanica/2104

DOI : 10.4000/germanica.2104

ISSN : 2107-0784

\section{Éditeur}

Université de Lille

\section{Édition imprimée}

Date de publication : 1 janvier 1992

Pagination : 199-206

ISSN : 0984-2632

\section{Référence électronique}

Erika Tunner, «La traduction, art second », Germanica [En ligne], 10 | 1992, mis en ligne le 03 avril 2014, consulté le 06 octobre 2020. URL : http://journals.openedition.org/germanica/2104 ; DOI : https:// doi.org/10.4000/germanica.2104

Ce document a été généré automatiquement le 6 octobre 2020.

(C) Tous droits réservés 


\title{
La traduction, art second
}

\author{
Die Kunst des Übersetzers
}

\author{
Erika Tunner
}

1 Beaucoup de traducteurs se disent modestement simples artisans. Philologues scrupuleux et avertis, conscients des problèmes théoriques et pratiques de la traduction, ils s'attachent à rendre la «langue de départ » dans la « langue de cible ", avec le moins d'écarts possibles par rapport au texte original. Lorsque les équivalents satisfaisants font défaut, lorsque pour le choix final un pis-aller s'impose, ils n'aiment pas trop entendre parler du lieu commun de la «trahison » mais constatent « une défaite ", regrettable certes, inévitable aussi. Ces traducteurs sont des hommes de métier. Leur souci principal est d'arriver au plus près du sens, Ils méritent le plus grand respect pour le sérieux de leur travail.

2 Anciennement, et jusqu'au milieu du XVIII ${ }^{\mathrm{e}}$ siècle, artisan se dit pour artiste. Le traducteur artisan qui excelle dans son art, est un artiste. Et si l'inspiration de l'artiste anime le traducteur, celui-ci se fait « passe-muraille ». Il est vrai que ces traducteurs ne sont pas toujours les plus fidèles. Le problème de la trahison leur est douloureusement familier. Il leur arrive de céder au sens du plaisir sans écouter la voix de la raison. Mais est-ce vraiment par manque de sagesse que l'on tente l'aventure? Et la fidélité restreinte au dévouement irréprochable mais inflexible, n'est-ce pas aussi une manière de trahison?

3 Les traducteurs-artisans, pour la plupart, ont tendance à dire que les harmonies imitatives n'enchantent que celui qui connait la signification des mots. Ils n'ont pas tout à fait tort : évidemment, les vers célèbres d'Alfred de Vigny «J'aime le son du cor, le soir, au fond des bois » suscitent d'autres impressions que la constatation banale « J'ai un cor au pied »...

4 Les traducteurs " passe-muraille » en revanche, croient à la magie des sonorités et des timbres, faisant moins appel aux connaissances linguistiques qu'à la disposition d'accueillir un ensemble d'éléments poétiques et musicaux sans en avoir une intelligence immédiate. Un non-germaniste ignorant tout de l'allemand, restera-t-il parfaitement insensible à l'écoute de cette petite berceuse de Clemens Brentano: 
Singt ein Lied so süß gelinde,

Wie die Quellen auf den Kieseln,

Wie die Bienen um die Linde

Summen, murmeln, flüstern, rieseln?

5

La syntaxe du quatrain est loin d'être d'une logique transparente. Mais le sens, dans un poème, n'est pas toujours son trait principal ni sa qualité première. Ici, quelqu'un nous demande de chanter une chanson douce, à voix basse. Puis il est question de sources qui «chuchotent et ruissellent " sur la grève, tandis que des abeilles «susurrent et murmurent» en tournant autour d'un tilleul. Mais par la place des virgules, par l'énumération des verbes à la fin du quatrain, ces rapports ne sont nullement évidents, ils sont même adroitement entremêlés : ce n'est pas le raisonnement qui domine mais la correspondance secrète entre les choses - et c'est elle qu'il s'agit de reproduire, de « ressusciter » dans la traduction :

Chantez un susurrement

de source sur la grève.

Chantez un murmure d'abeille

qui bruinent au tilleul

(René Lasne) double parfait, c'est s'engager sur le chemin de l'impossible. Mais les vers français laissent passer la lumière de l'original sans permettre d'en distinguer nettement tous les contours.

D'autre part, il existe une manière de traduire qui se révèle être le point de départ d'une création toute personnelle et en grande partie indépendante de l'original. C'est un «cas-limite » de la traduction, en fait peut-être le plus intéressant, dans la mesure où l'auteur, s'inspirant d'une œuvre d'art, en produit une variante qui exprime son univers à lui.

8 Le premier grand traducteur allemand est un artisan-artiste à la fois d'une grande puissance verbale et d'une étonnante érudition. Désireux de rendre la lecture de Rabelais agréable au public allemand, il commence par traduire Gargantua aussi fidèlement qu'il le peut. Puis son propre génie s'enflamme et l'emporte : procédant d'abord à quelques transformations, abrégeant plusieurs chapitres et les fondant ensemble, il finit par prendre la parole lui-même pour exposer ses propres idées, dans un langage savoureux, truculent, digne de Rabelais mais chargé d'une intention moralisante que l'on chercherait en vain dans les statuts de l'abbaye de Thélème... L'œuvre née ainsi, publiée en 1575, puis en 1782, Fischart l'appelle d'abord Geschichtschrift (Écrit d'histoire) mais, jugeant ce titre trop sérieux et trop peu conforme à sa façon de travailler, il préfère en fin de compte le terme de Geschichtsklitterung, « Histoire écrite à la hâte », « Histoire barbouillée ».

9 Plus proche de notre époque se situe le «destin » d'une ballade qui évoque la figure d'une femme "damnée ", victime de son propre maléfice. Il s'agit de la célèbre Loreley, créée de toutes pièces par Clemens Brentano. Le poème fait partie du roman Godwi, publié à la fin de 1801. Le point de départ : un rocher sur le Rhin, connu pour son écho, mais où la légende locale n'avait nullement placé une femme fatale. Dans la ballade de Brentano, Lore Lay (c'est sous cette forme que son nom apparaît pour la première fois) est une jeune fille de condition modeste qui vit dans le cadre médiéval de la petite cité de Bacharach. La fascination qu'elle exerce sur les hommes vient de son regard, non de son chant ni de sa blonde chevelure comme plus tard chez Heine. Accusée de sorcellerie et traînée devant l'évêque qu'elle subjugue malgré elle, elle se voit condamnée au 
cloître et non pas au bûcher qu'elle implore. Sur le chemin vers le couvent, elle réussit à échapper à la surveillance des chevaliers chargés de l'accompagner; elle grimpe sur un haut rocher et se précipite dans le Rhin où elle avait cru apercevoir la barque de son amant infidèle, le seul qu'elle eût aimé et qui eût résisté à son pouvoir. Pendant longtemps, le poème de Brentano resta pratiquement inconnu. Mme de Staël ne le signale pas dans son livre sur l'Allemagne. Hugo, Nerval et bien d'autres encore ne procèdent pas de Brentano mais du professeur polygraphe, Aloys Schreiber, que Brentano avait rencontré à Heidelberg. De bonne heure, Schreiber s'est spécialisé dans un genre qui allait connaître un essor prodigieux à partir de 1820 environ : les guides de voyage. Son Guide du Rhin dans lequel il rapporte la «légende» de la Loreley, fut traduit en français à plusieurs reprises et était un constant livre de référence.

Deux poètes français sont revenus à la source de Brentano, mais avec une discrétion extrême ils ne mentionnent même pas son nom : Jean Lorrain qui publie en 1898 un curieux poème en prose, Loreley, dans le style fin-de-siècle, et Guillaume Apollinaire qui, en 1902, fait une traduction de la ballade de Brentano. Les cinq premières strophes donnent d'emblée une idée des procédés de traduction qui sont à l'origine de la version d'Apollinaire : la traduction littérale, l'adaptation libre, la fusion de plusieurs éléments en un seul.

\section{Brentano :}

Zu Bacharach am Rheine

Wohnt' eine Zauberin,

Sie war so schön und feine

Und riss viel Herzen hin.

Und machte viel zu schänden

Der Männer ringsumher,

Aus ihren Liebesbanden

War keine Rettung mehr.

Der Bischof Hess sie laden

Vor geistliche Gewalt

Und musste sie begnaden

So schön war ihr' Gestalt.

Er sprach zu ihr gerühret;

«Du arme Lore Lay!

Wer hat dich denn verführet

Zu böser Zauberei?»

« Herr Bischof, lasst mich sterben

Ich bin des Lebens müd'

Weil jeder muss verderben,

Der meine Augen sieht».

Apollinaire :

A Bacharach, il y avait une sorcière blonde

Qui laissait mourir d'amour tous les hommes à la ronde

Devant son tribunal l'évêque la fit citer -

D'avance il l'absolvit à cause de sa beauté.

o belle Loreley aux yeux pleins de pierreries, De quel magicien tiens-tu ta sorcellerie? 
Je suis lasse de vivre et mes yeux sont maudits

Ceux qui m'ont regardée, évêque, en ont péri.

11 Apollinaire réunit deux vers en un et fait du quatrain un distique, gardant cependant quelque chose de la métrique des vers allemands puisqu'il est difficile de changer les distiques en quatrains. Les modifications du sens sont plus importantes. Nulle part, chez Brentano, il n'est question de la chevelure blonde de la Lore Lay. Il faut attendre Heine - qu'Apollinaire avait lu - pour trouver cet attribut de Beauté qui dorénavant comptera, ainsi que la voix enchanteresse, parmi les « signes distinctifs » de la Loreley. Apollinaire n'insiste pas, expressis verbis, sur l'émotion de l'évêque (Brentano: «Er sprach zu ihr gerühret») : plus intériorisée, cette émotion s'exprime dans les paroles mêmes que l'évêque adresse à Lore, avec plus de franchise ou tout simplement avec plus de spontanéité que chez Brentano. Chez Apollinaire, l'évêque est d'emblée ému par la beauté de la jeune fille ("O belle Loreley ») et ne s'en cache pas. Chez Brentano, il est (ou se dit) ému par sa souffrance («du arme Lore Lay») mais la suite du poème n'exclut pas une pratique de dissimulation, voire d'hypocrisie chez cet homme d'Église. A la fin du poème de Brentano, Lore Lay, comme nous l'avons dit, grimpe sur un rocher, aperçoit une barque, croit y reconnaître son amant, «se penche alors et tombe dans le Rhin » («Da lehnt sie sich hinunter/ Und stürzet in den Rhein»). L'ambiguïté qui subsiste dans les vers allemands entre la décision du suicide et le hasard d'un accident n'échappera pas à un lecteur attentif, et Apollinaire la conserve dans sa traduction. Mais Apollinaire supprime les deux dernières strophes de Brentano qui évoquent la mort des chevaliers accompagnant Lore Lay au couvent, et qui font par ailleurs allusion à l'écho célèbre du rocher au bord du Rhin. Il les remplace par une tout autre vision de la figure de la Lore Lay et de sa disparition :

Mon cœur devient si doux, c'est mon amant qui vient.

Elle se penche alors et tombe dans le Rhin

Pour avoir vu dans l'eau la belle Loreley

Ses yeux couleur du Rhin, ses cheveux de soleil.

\section{«Pour avoir vu dans l'eau la belle Loreley »...}

Elle ne meurt donc pas, ou pas seulement, d'un cœur brisé ; elle ne meurt pas, ou pas seulement, parce que son regard au pouvoir magique fait périr les hommes. Elle meurt comme ensorcelée d'elle-même, amoureuse de sa propre image, éprise de sa propre beauté, se complaisant en elle-même, se choisissant comme objet érotique, indifférente à toute autre personne, à toute autre chose : «Ich liebe keinen mehr» («je n'aime plus personne »), dit-elle chez Brentano, et Apollinaire renchérit : «Je n'aime rien ». Ce côté narcissique de la Loreley d'Apollinaire ne se trouve pas dans la ballade allemande, à moins qu'on ne veuille voir une allusion d'une discrétion raffinée dans la strophe que voici :

Ich will noch einmal sehen

Wohl in den tiefen Rhein

Und dann ins Kloster gehen

Und Gottes Jungfrau sein.

(Je voudrais jeter un dernier regard

dans le Rhin profond

puis j'irai au couvent

et me ferai religieuse). 

tombé dans l'oubli. Il en est tout autrement de la traduction du poème de Brentano qu'un certain F. Delacroix a publié à Paris en 1843, dans un recueil intitulé Fleurs d'Outre Rhin (éd. Charpentier) ;

Ralentis un peu ta vitesse

O Rhin ! de Bacharach voici les bords charmants

C'est ici qu'autrefois pour plaire à mille amants

Vécu certaine enchanteresse.

Un maléfice des enfers

Créa-t-il sa beauté qui perdit bien des hommes?

Nul du moins ne pouvait, dans les lieux où nous sommes,

Se croire à l'abri de ses fers.

Pour elle préparant sa foudre,

Au tribunal sacré l'évêque l'appela ;

Et, tant elle était belle, à peine elle fut là,

Qu'il ne songea plus qu'à l'absoudre !

Le cœur ému, mais sans délai

Par trois fois se signant pour que Dieu le protège :

- Qui donc su t'enseigner magie et sortilège ?

Réponds... ô pauvre Loreley! -

Bien mal je défendrai ma vie,

Oh ! de grâce, à juger vous qu'on dit très expert,

Sire Évêque, ordonnez qu'elle me soit ravie.

Qui m'ose regarder se perd.

À travers le prisme de la traduction de F. Delacroix - lui aussi tait le nom de l'auteur allemand - on peut certes apercevoir le poème original. Comme Apollinaire, F. Delacroix introduit des modifications mais contrairement à Apollinaire il ne raccourcit pas, il ne condense pas : il allonge, il dilue. S'adressant au Rhin, sur le ton de l'amitié, il lui demande de ralentir un peu sa vitesse, il évoque des bords charmants dont Brentano ne nous dit rien. S'il garde la miséricorde, vraie ou fausse, que l'évêque manifeste à l'égard de la Loreley, il ajoute que l'évêque se signe, sans délai et par trois fois, et qu'il juge bon de nous en donner la raison: pour que Dieu le protège... Systématiquement, l'original est rationnalisé. Le style apparemment simple et naïf de Brentano (savamment simple et naïf) s'est transformé en style soutenu. Chez Brentano, il $\mathrm{y}$ a une différence très nette entre le langage de Lore Lay qui n'a pas appris son allemand dans une Grande École, et celui de l'évêque, homme cultivé et mondain. Cette différence a totalement disparu chez F. Delacroix : Loreley et l'évêque s'expriment avec la même élégance, n'ignorant rien du «bon usage » du français. Dans une certaine mesure, Delacroix dénature l'esprit de l'original.

Mais chaque traduction est bien évidemment aussi une lecture d'un poème, par une personne précise, dans une époque donnée. D’une façon plus générale : au moment où 
nous lisons un texte, nous le traduisons déjà pour nous-mêmes avant de lui donner une forme nouvelle, dans une autre langue. Inévitablement, le traducteur, lui aussi, se communique et se fait connaître à travers « sa » traduction.

\section{RÉSUMÉS}

Le traducteur est à la fois artisan et artiste. Quels sont ses plaisirs et ses difficultés, ses réussites et ses échecs? Les «faux monnayeurs » de la traduction ne se trouvent-ils pas en fait parmi les traducteurs-écrivains qui nourrissent leurs propres œuvres de celles qu'ils traduisent? Mais après tout, n'est-ce pas précisément un des éléments les plus séducteurs du travail de la traduction? Nous essayons de répondre à ces questions par un exemple concret : la ballade de la Lore Lay de Brentano et ses adaptations françaises.

Traduire, on le sait, est une entreprise impossible. Mais l'impossible, de tout temps, est une aventure qui nous tente.

Der Übersetzer ist zugleich Handwerker und Künstler. Welches sind die Freuden und Leiden des Übersetzens, die geglückten und die gescheiterten Versuche? Findet man die «Falschmünzer» der Übersetzer nicht gerade unter jenen, die selbst Schriftsteller sind und die aus dem fremden Werk die Substanz für das eigene ziehen? Doch ist dies nicht wiederum der eigentlich verführerische Aspekt beim Übersetzen? Wir versuchen, auf diese Fragen mit einem konkreten Beispiel zu antworten: mit der Ballade von Brentanos Lore Lay und ihren Übertragungen ins Französische.

Übersetzen ist bekanntlich ein unmögliches Unterfangen. Aber seit eh und je geht von dem Abenteuer des Unmöglichen der größte Reiz aus.

\section{AUTEUR}

\section{ERIKA TUNNER}

Université Paris XII 